\title{
ARTICLE
}

\section{Analysis of reactor-neutrino oscillation experiment based on consideration of internal constituent motion}

\author{
Kenji Ishibashi ${ }^{\mathrm{a}, \mathrm{b}}$, Shoya Suda ${ }^{\mathrm{a}^{*}}$, Eunji Lee ${ }^{\mathrm{a}}$ and Nobuhiro Shigyo ${ }^{\mathrm{a}}$ \\ ${ }^{a}$ Department of Applied Quantum Physics and Nuclear Engineering, Kyushu University, 744 Motooka, Nishi-ku, Fukuoka, \\ 819-0395, Japan; ${ }^{b}$ Center for Accelerator and Beam Applied Science, Kyushu University, 744 Motooka, Nishi-ku, Fukuoka, \\ 819-0395, Japan
}

Experimental data of neutrino oscillation have been obtained for electron antineutrinos from nuclear reactors in recent years. The experimental data have been successfully analyzed by assuming that the oscillation phenomenon is expressed by mixing angle $\theta_{i j}$ and squared eigen neutrino mass difference $\Delta m_{i j}^{2}$. It is of interest to explain the phenomena from another point of view. We attempted to find the neutrino internal motion that is consistent to the standard theory for weak interaction. As a test of internal constituent motion, the time evolution of internal particles was calculated. It was found that the oscillation length of $100 \mathrm{~km} \mathrm{level}$ was explainable without use of $\Delta m_{i j}^{2}$. A discussion was provided about possible existence of additional constituent in the neutrino system.

\section{Keywords: electron neutrino; weak interaction; internal constituent; reversed-type auxiliary field}

\section{Introduction}

Nuclear reactors emit a great number of electron antineutrinos $\left(\sim 10^{21}\left[\mathrm{~s}^{-1}\right]\right.$ for typical $\left.1 \mathrm{GW}-\mathrm{PWR}\right)$. The standard theory for electroweak interaction contains two important features: (1) Potential interaction in group theory of $\mathrm{U}(1) \times \mathrm{SU}(2)$ frame [1-3], and (2) Higgs field for mass generation and use of massive weak bosons $[4,5]$. The standard theory has been successfully applied to description of many weak interaction reactions [6].

In recent years, experimental data of neutrino oscillation for electron antineutrinos from nuclear reactors have been obtained, following to atmospheric and accelerator neutrino experiments. Experimental data on electron neutrinos are analyzed on the basis of PMNS matrix [7,8]: flavor eigen state is expressed by mixing angle $\theta_{i j}$ and intrinsic neutrino mass state $m_{i}$. The KamLAND collaboration for electron antineutrino provided $\Delta m_{21}^{2} \approx 7.5 \times 10^{-5} \mathrm{eV}^{2}$, giving an oscillation length of $L=(\pi / 1.27) E / \Delta m_{21}^{2} \approx 100 \mathrm{~km}$ for neutrino energy $E=3 \mathrm{MeV}$ [9]. Meanwhile, recent Daya-Bay, Reno, and D-Chooz collaborations presented $\Delta m_{31}^{2} \approx 2.45 \times 10^{-3} \mathrm{eV}^{2}$ and $L \approx 3 \mathrm{~km}$ for the same energy [9].

It is noted that the neutrino is treated as a single structure-less particle in both treatments for neutrino interaction cross sections and neutrino oscillations. The experimental results on neutrino oscillation are supposed

\footnotetext{
*Corresponding author.Email: kisibasi@kune2a.nucl.kyushu-u.ac.jp
}

to contain some information on internal motion, but no investigation has been made from this point of view.

We attempt to find the neutrino internal motion, which predicts the oscillation length in a manner being consistent to the above two features of (1) and (2). Our group previously took experimental data near nuclear reactor core [10]. The data are not explained by the standard theory at all. For this reason, we will further discuss about possible inclusion of an additional constituent, to which the reaction mechanism for the experimental data may be ascribed.

\section{Proposed internal constituent motion being consistent to electroweak theory}

The standard theory includes two dominant features (1) and (2) described above. For the potential interaction (1), the Weinberg angle $\theta_{W}$ determines the relative strength of $U(1)$ and $S U(2)$ interactions in an overall manner. Use of $\sin ^{2} \theta_{W}=0.231$ [6] gives coupling constant $g=e / \cos \theta_{W}=1.14 e$ for $\mathrm{U}(1)$ for directional hypercharge $\mathrm{Y}$ interaction, and $g^{\prime}=$ $e / \sin \theta_{W}=2.08 e$ for $\mathrm{SU}(2)$ for rotational interaction. Since internal constituents should follow Dirac-type Equation with Vector (V) and Axial Vector (AV) matrices, they are possible to own either positive or negative mass. If the hypercharge works with exactly the same size of $e$, it is natural for neutrino constituents to have two hypercharges and three hypercharge moments with mixed 
Table 1. Assignment of integer number of $e$ to be consistent to coupling constants with Weinberg angle $\theta_{W}$.

\begin{tabular}{|c|c|c|}
\hline & $\begin{array}{l}\text { Hypercharge } \\
\text { type } \\
\quad \mathrm{U}(1), Y \\
g=1.14 e<2 e\end{array}$ & $\begin{array}{l}\text { Moment type } \\
\operatorname{SU}(2), \sigma \\
g^{\prime}=2.08 e<3 e\end{array}$ \\
\hline Positive mass & $1 e_{1}$ & $1 e_{1}$ \\
\hline Negative mass & $\bar{e}$, & $\bar{e}$ \\
\hline
\end{tabular}

mass polarity. The situation is explained in Table $\mathbf{1}$. Existence of mixed polarity charge reduces the effective number of hypercharge and hypercharge moment in the electroweak theory.

The feature (2) is related to the covariant properties of momentum and potential in the Dirac-type equation. The constituent mass $m_{v}$ is obtained as eigen value of a resultant overall $4 \times 4$ matrix. Time-direction momenta in $\mathrm{V}$ - and AV-types should be canceled out to produce a scalar value of mass. We assume that $4 \times 4$ gamma matrix $\gamma^{v}$ retains the function of transforming four-momenta in a conventional space $U^{c}$ into those in its own special space $U^{v}$. We consider four types of constituent particles $v=0-3$ corresponding to four basic gamma matrices $\gamma^{0-3}$. When the gamma matrix $\gamma^{v}$ converts momentum $p^{\mu}$ from space $U^{c}$ to $U^{v}$, one of momenta $p^{\kappa_{v}}$ at $U^{c}$ is converted into time-directional momentum $p^{0\left(\kappa_{v}\right)}$ at $U^{v} ; \kappa_{v}=$ $0,3,3,2$ for $v=0,1,2,3$. For usual electromagnetic system, auxiliary field $B_{\mathrm{X}}=-\partial_{\mu, \mathrm{X}} A_{\mathrm{X}}^{\mu}(\mathrm{X}=\mathrm{V}$ or $\mathrm{A})$ is defined [11], but such field generates no actual energy by coupling with specified velocity and leads to no effect to potential interaction. The $\kappa_{v}$-direction potential in AV type is assigned to have the time-like property, and to produce a reversed-type auxiliary field $B_{\text {revA }}=-i \partial_{\kappa_{v}, \mathrm{~A}} A_{\mathrm{A}}^{0\left(\kappa_{v}\right)}$. Since $B_{\text {revA }}$ couples with the $\kappa_{v}$-direction motion even in $U^{c}$, it produces an actual energy, which should work as neutrino constituent mass as explained later. Since the field $B_{\text {revA }}$ is defined in the frame of $\mathrm{U}(1) \times \mathrm{SU}(2)$, it works as Higgs-like mass-generation field.

As for the oscillation length, the internal constituents are considered to be bound and move around the center of mass of neutrino system in an oscillational manner.

The basic formulation for internal constituent motion is given as follows. The Lagrangian density of the system is written by the sum of individual constituents $v$ as

$$
\begin{aligned}
& \mathcal{L}=\sum_{v} \mathcal{L}_{v} \\
& \mathcal{L}_{v}=\bar{\psi}_{\nu}\left\{\left(i \gamma^{\mu} D_{\mu, \mathrm{V} v}\right)+\gamma^{5}\left(i \gamma^{\mu} D_{\mu, \mathrm{A} v}\right)-m_{v}\right\}_{k i n} \psi_{\nu} \\
& +\left\{-F_{\mu \pi, \mathrm{V} v} F_{\mathrm{V} v}^{\mu \pi} / 4+\left(B_{\mathrm{V} v}\right)^{2} / 2+B_{\mathrm{V} v} \partial_{\mu} A_{\mathrm{V} v}^{\mu}\right\}_{\text {potv }} \\
& +\left\{-F_{\mu \pi, \mathrm{A} v} F_{\mathrm{A} \nu}^{\mu \pi} / 4+\left(B_{\mathrm{A} v}\right)^{2} / 2+B_{\mathrm{A} \nu} \partial_{\mu} A_{\mathrm{A} v}^{\mu}\right\}_{\text {potA }} \\
& D_{\mu, \mathrm{V} v}=\partial_{\mu, \mathrm{V} v}+Q_{v} i A_{\mu, V v} \\
& D_{\mu, \mathrm{A} v}=\left\{\begin{array}{ll}
\partial_{\mu, \mathrm{A} v}+Q_{\nu} i A_{\mu \neq \kappa_{v}, \mathrm{~A} v} & \mu \neq \kappa_{v} \\
\partial_{\kappa_{v}, \mathrm{~A} v}+Q_{v} d_{\nu} i B_{\mathrm{rev} \mathrm{A} v} & \mu=\kappa_{v}
\end{array},\right.
\end{aligned}
$$

where Feynman gauge is adopted, and $d_{v}$ is a characteristic length of constituent $v$. The eigenvalue in the above kinetic term with regards to gamma matrices produces a kinetic mass $m_{v}$. It is noted that $\left(p_{\mathrm{Av}}^{\kappa_{v}}-\right.$ $\left.Q_{v} d_{v} B_{\mathrm{revA} \nu}\right)^{2}$ is separated into $\left(p_{\mathrm{Av}}^{\kappa_{v}}\right)^{2}+\left(Q_{v} d_{v} B_{\mathrm{revA}}\right)^{2}$ due to difference in real and imaginary values. Since the time component in $U^{v}$ requests that $p_{\mathrm{V} v}^{\kappa_{v}}-Q_{v} A_{\mathrm{V} v}^{\kappa_{v}}$ is treated to be canceled out with $p_{\mathrm{Av}}^{\kappa_{v}}$ in a squared form of the same covariant quantity, the remaining part of squared mass $\left(m_{v}\right)^{2}$ meets

$$
\left(m_{v}\right)^{2}=\left(Q_{v} d_{v} B_{\mathrm{revA} v} / i\right)^{2} \equiv\left(Q_{v} d_{v} B_{\mathrm{revA} v}^{0}\right)^{2}
$$

Difference between $\mathrm{V}$ and $\mathrm{AV}$ field energy terms in $\mathcal{L}_{v}$ gives the magnetic field energy in $\mathrm{AV}$ spin, i.e. self-energy $E_{\mathrm{A} v}^{\mathrm{spin}}$. Therefore, $m_{v}$ should be equal to $E_{\mathrm{A} v}^{\text {spin }}$. The two energies in $\mathcal{L}_{v}$ need to satisfy

$$
\left|m_{v}\right|=E_{\mathrm{A} v}^{\text {spin }} \text {. }
$$

Dirac-type Equation is given for each constituent $v$ as

$$
\left\{\left(i \gamma^{\mu} D_{\mu, \mathrm{V} v}\right)+\gamma^{5}\left(i \gamma^{\mu} D_{\mu, \mathrm{Av}}\right)-m_{v}\right\} \psi_{v}=0
$$

The mass $m_{v}$ is taken to have time-directional gamma matrix and be substituted by $\gamma^{0} m_{v}$ in actual calculation. The value of $m_{v}$ is given as eigen value for $4 \times 4$ matrix defined by the above equation. The total mass is given by linear sum as

$$
m_{s}=\sum_{v} m_{v}
$$

The value $m_{v}$ is mass with plus or minus polarity for linear expression by Dirac-type equation. The hypercharge of constituent $v$ is given for potential interaction in the linear system by

$$
Q_{v}=\mathrm{e} m_{v} / m_{s} .
$$

Dirac-type equation produces motion of separately moving multibody constituents like electrons in an atom. However, neutrino system mass is considered to be concentrated as if it is one body. For this reason,

$$
\left(m_{s}\right)^{2}=\sum_{v}\left(m_{v}^{\text {int }}\right)^{2}
$$

where $m_{v}^{\text {int }}$ is intrinsic mass with either real or pure imaginary quantity. The relation $m_{v}=\left(\mathrm{m}_{v}^{\text {int }}\right)^{2} / m_{s}$ keeps the consistency between Eqs. (2) and (3). Eq. (3) gives

$$
m_{s}=\left\{\Sigma_{v}\left(m_{v}^{\text {int }}\right)^{2}\left(\dot{x}_{k, \mathrm{~V} v} \dot{x}_{\mathrm{V} v}^{k}-\dot{x}_{k, \mathrm{~A} v} \dot{x}_{\mathrm{A} v}^{k}\right)\right\}^{1 / 2}
$$

with relativistic relation $\dot{x}_{k, \mathrm{~V} v} \dot{x}_{\mathrm{V} v}^{k}-\dot{x}_{k, \mathrm{~A} v} \dot{x}_{\mathrm{A} v}^{k}=1$. One can see that the whole $\mathrm{V}$ motion takes a role of time motion, while AV movement works as space-like motion. Total energy $E$ is constructed by correcting mass polarity as well as $\mathrm{V}$ and $\mathrm{AV}$ polarity in the above as

$E=\left[\sum_{v}\left|\frac{m_{s}}{m_{v}}\right|\left\{\begin{array}{c}\left(p_{\mathrm{V} v}^{k}-e A_{\mathrm{V} v}^{k}\right)\left(p_{\mathrm{V} v}^{k}-e A_{\mathrm{V} v}^{k}\right) \\ +\left(p_{\mathrm{A} v}^{k \neq \kappa_{v}}-e A_{\mathrm{A} v}^{k \neq \kappa_{v}}\right)\left(p_{\mathrm{A} v}^{k \neq \kappa_{v}}-e A_{\mathrm{A} v}^{k \neq \kappa_{v}}\right) \\ +\left(p_{\mathrm{Av}}^{\kappa_{v}}\right)^{2}+\left(Q_{v} d_{v} B_{\mathrm{revA} \nu}^{0}\right)^{2}\end{array}\right\}\right]^{1 / 2}$

After momentum $p_{X v}^{k}$ is converted into $p_{X v E}^{k}=$ $\left(m_{s} / E\right) p_{X v}^{k}$ for the canonical conjugate momentum for total energy, the expression serves as Hamiltonian.

The constituent motions are derived from canonical equations according to usual procedure. In the calculation, Lagrange multiplier method is utilized: the relativistic relation is explicitly imposed by 


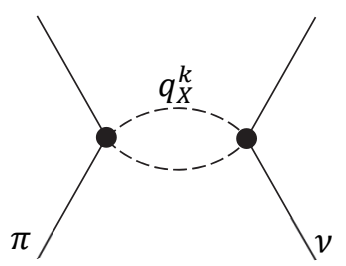

Figure 1. Potential interaction through momentum transfer $q_{X=\mathrm{V}, \mathrm{AV}}^{k}$ between two particles $v$ and $\pi$.

$$
\begin{aligned}
& \lambda_{v}^{(1)}\left\{\dot{x}_{\mathrm{V} v}^{k} \dot{x}_{\mathrm{V} v}^{k}-\dot{x}_{\mathrm{A} v}^{k} \dot{x}_{\mathrm{A} v}^{k}-1\right\}_{k=\kappa_{v}}, \\
& \lambda_{v}^{(2)}\left\{\dot{x}_{\mathrm{V} v}^{k} \dot{x}_{\mathrm{V} v}^{k}-\dot{x}_{\mathrm{A} v}^{k} \dot{x}_{\mathrm{A} v}^{k}\right\}_{k \neq \kappa_{v}} .
\end{aligned}
$$

Potential propagator in the case of $Q$ to $Q$ interaction is assumed to be simply written by

$$
\begin{aligned}
& Q_{\pi} \frac{1}{q_{\mathrm{V}}^{k} q_{\mathrm{V}}^{k}-q_{\mathrm{A}}^{k} q_{\mathrm{A}}^{k}} Q_{\nu}=e \frac{1}{m_{s}^{2}} e, \\
& Q_{v}=e m_{v} / m_{s} \text { and } q_{\mathrm{V}}^{k} q_{\mathrm{V}}^{k}-q_{\mathrm{A}}^{k} q_{\mathrm{A}}^{k}=m_{\pi} m_{v}
\end{aligned}
$$

where $m_{s}$ is again the total mass of neutrino system. The Feynman graph is shown in Figure $\mathbf{1}$ for potential propagation. The product of mass $m_{s}$ and coefficient $\lambda_{v}^{(1)}$ is considered to be close to $Z^{0}$ mass.

\section{Calculation example}

The motion of constituents was calculated during variation of the intrinsic time $c \tau(\mathrm{fm})$ which was set on the neutrino system. The canonical equation was solved for the Hamiltonian expressing the total energy in Eq. (4). During time evolution, constituent masses are determined self-consistently to meet Eq. (1). The original Lagrangian density $\mathcal{L}_{v}$ indicates that the $\mathrm{V}$ and AV motions work as time and spatial movements as a whole. However, no implicit relation guaranties that the neutrino system continuously flies with almost complete light velocity. The Lagrange multipliers $\lambda_{v}^{(1)}$ and $\lambda_{v}^{(2)}$ are determined to make the brackets of Eqs. (5) and (6) zero. We found that achievement of the relativistic relation requires quite large values of the order of $\lambda_{v}^{(1)} \sim 10^{15}$ : The products $m_{s} \lambda_{v}^{(1)}$ reaches about 100 $\mathrm{GeV}$ of $Z^{0}$ boson mass [6]. For this reason, we simply set at $\lambda_{v}^{(1)}=m_{Z^{0}} / m_{s}$.

In actual calculation, the initial positions of constituents were taken to all reside on the $z$ axis for simplicity. The positions and velocities as well as masses were random sampled at $c \tau=0$.

As reference values of masses, a principal constituent particle 1 of hypercharge type was set to have Compton length $\lambda_{c}=1 /\left|m_{v=1}^{i n t}\right|$ for the purpose of reproducing oscillation length of $3 \mathrm{~km}$ for $3 \mathrm{MeV}$ neutrino.

Two types of calculation examples are listed in Table 2 for individual constituents. In the table, particles +1 and 0 in case 1 reside in the positive mass state, and they change into the negative one in case 2. Similarly, particles -1 and 2 in the negative mass state in case 1 vary into the positive one in case 2 .

Two examples are presented in Figure 2 for positions in time evolution. The motions in case 1 stably continue from $c \tau=-3000$ to $3000 \mathrm{fm}$. Beyond this range, the solution gets unstable and jumps to another state. The range corresponds to a flight length of $110 \mathrm{~km}$. However, in case 2, the duration was stable from -2000 to $2000 \mathrm{fm}$. The stable duration range depends to some extent on initial value of $\lambda_{v}^{(2)}$.

One can see that the constituents move basically in reversed direction in cases 1 and 2 . Therefore, they take vibration motion around the moving center of mass. The motions of two cases are considered to constitute vibration motion, where mass polarity varies according to velocity change. The oscillation length is $91.5 \mathrm{~km}$ in average for the example.

Table 2. Examples of two solutions (cases 1 and 2). V- and $\mathrm{AV}$-wave functions are assigned orthogonally with antisymmetrization by two spherical harmonics. The radial function takes a Gaussian form with radial standard deviation parameter.

\begin{tabular}{llccc}
\hline \multicolumn{4}{c}{ Case 1 } & (motion in original direction around c.m.) \\
\hline Positive & Transform. $\gamma^{v}$ & $\gamma^{1}$ & $\gamma^{0}$ & $\gamma^{3}$ \\
mass state & Particle no. & +1 & 0 & 3 \\
(Helic.-) & Spher. Harmo. & $Y_{2}^{-1} \& Y_{2}^{-2}$ & $Y_{1}^{-1} \& Y_{2}^{-1}$ & $Y_{1}^{0} \& Y_{2}^{0}$ \\
\hline Negative & Transform. $\gamma^{v}$ & $\gamma^{1}$ & $\gamma^{2}$ \\
mass state & Particle no. & -1 & 2 \\
(Helic.+) & Spher. Harmo. & $Y_{2}^{1} \& Y_{2}^{2}$ & $Y_{1}^{1} \& Y_{2}^{1}$ \\
\hline
\end{tabular}

\begin{tabular}{llccc}
\hline \multicolumn{5}{c}{ Case 2 (basically in reversed direction around c.m.) } \\
\hline Positive & Transform. $\gamma^{v}$ & $\gamma^{1}$ & $\gamma^{2}$ & $\gamma^{3}$ \\
mass state & Particle no. & +1 & 2 & 3 \\
(Helic. - ) & Spher. Harmo. & $Y_{2}^{-1} \& Y_{2}^{-2}$ & $Y_{1}^{-1} \& Y_{2}^{-1}$ & $Y_{1}^{0} \& Y_{2}^{0}$ \\
\hline Negative & Transform. $\gamma^{v}$ & $\gamma^{1}$ & $\gamma^{0}$ \\
mass state & Particle no. & -1 & 0 \\
(Helic. + ) & Spher. Harmo. & $Y_{2}^{1} \& Y_{2}^{2}$ & $Y_{1}^{1} \& Y_{2}^{1}$ \\
\hline \multicolumn{5}{c}{} \\
\hline Particle no. & \pm 1 & $0 / 2$ & 3 \\
$\left|m_{\nu}^{\text {int }}\right|(\mathrm{meV})$ & 8.88 & 6.59 & 0.0902 \\
Radius $\left(10^{6} \mathrm{fm}\right)$ & 2.48 & 3.83 & 304 \\
\hline
\end{tabular}
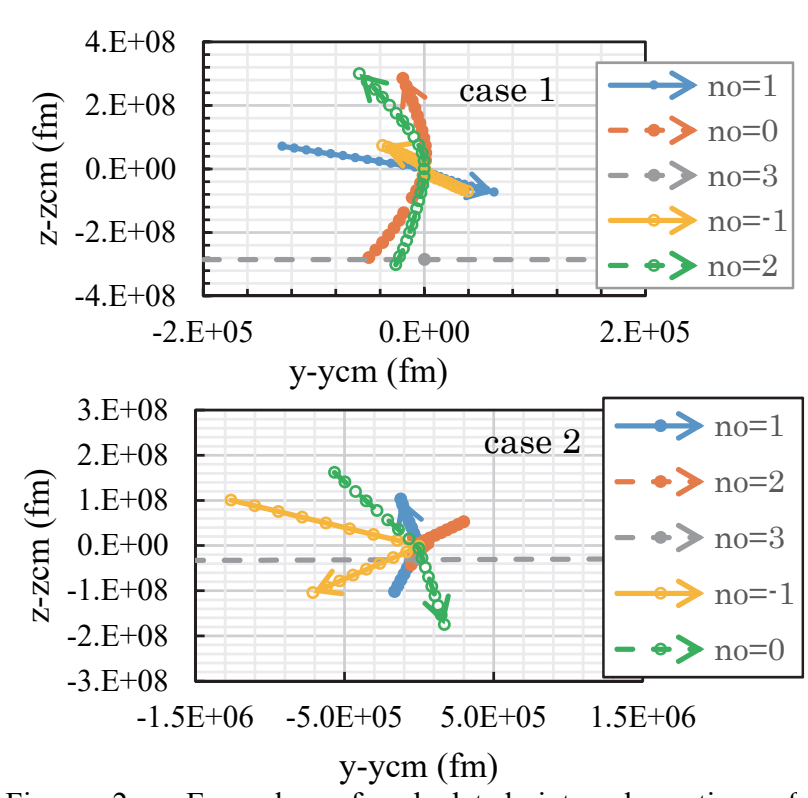

Figure 2. Examples of calculated internal motion of constituents in cases 1 and 2 . 


\section{Discussion on additional constituent particle}

The above allocation of internal constituents is understood to present a basic assignment. There may be another allocation, which includes an additional negative particle as a balanced mass distribution. The assignment is listed in Table 3. The generation of additional particle realizes three positive and three negative mass states, and accordingly reduces the total mass $m_{s}$. The additional particle (called no. 4) should have zero angular momentum, being admitted to have orbital (V and $\mathrm{AV}$ ) angular momentum $-1 / 2$ and spin $1 / 2$ in $z$-direction.

Since four gamma matrices were already utilized, the particle 4 is free from imposition of momentum-transformation gamma matrix and resides only in conventional space $\mathrm{U}^{c}$. For this reason, the particle is capable of neither generating nor receiving $B_{\mathrm{rev} A}^{0}$ to/from other particles. For the particle spherical harmonic function $Y_{0}^{0} \& Y_{1}^{-1}$, the effective values of $\mathrm{V}$ and AV angular momenta $l^{Z}$ are both $-1 / 4$. The $l_{A}^{Z}=$ $-1 / 4$ in AV type state is acceptable as negative mass state, since the $z$-motion of hypercharge-type particle -1 generates potential in time direction, and is inhibited from making interaction with particle 4 with $l_{A}^{z}=-1 / 4$. Therefore the spin of particle 4 should work as V type, instead of AV type. When the neutrino travels in an external V-type magnetic field like interplanetary magnetic field and geomagnetic one, the $\mathrm{V}$ type spin is capable of stably coupling with the magnetic field.

Since the particle 4 behaves differently from other constituents due to insusceptibility to $B_{\text {revA }}^{0}$, the additional particle may readily either disappear or separated due to external potential disturbance like $B_{\text {revA }}^{0}$. The experimental results by small electrochemical detector are supposed to be caused by incidence of such neutrinos (environmental neutrinos). It is considered to be possible for the additional particle with V-type spin to be dropped from the substantial part of neutrino and to couple with electron in water solution to make an electron with bosonic feature.

Table 3. Example of solution containing additional internal constituent particle.

\begin{tabular}{|c|c|c|c|c|}
\hline \multicolumn{5}{|c|}{ Case 1 (motion in original direction around c.m.) } \\
\hline \multirow{4}{*}{$\begin{array}{l}\text { Positive } \\
\text { mass state }\end{array}$} & Transform. $\gamma^{v}$ & $\gamma^{1}$ & $\gamma^{0}$ & $\gamma^{3}$ \\
\hline & Particle no. & +1 & 0 & 3 \\
\hline & Spher. Harmo. & $Y_{2}^{-1} \& Y_{2}^{-2}$ & $Y_{1}^{-1} \& Y_{2}^{-1}$ & $Y_{1}^{0} \& Y_{2}^{0}$ \\
\hline & Helicity & - & - & - \\
\hline \multirow{4}{*}{$\begin{array}{l}\text { Negative } \\
\text { mass state }\end{array}$} & Transform. $\gamma^{v}$ & $\gamma^{1}$ & $\gamma^{2}$ & Spin L_V \\
\hline & Particle no. & -1 & 2 & 4 \\
\hline & Spher. Harmo. & $Y_{2}^{1} \& Y_{2}^{2}$ & $Y_{1}^{1} \& Y_{2}^{1}$ & $Y_{0}^{0} \& Y_{1}^{-1}$ \\
\hline & Helicity & + & + & - \\
\hline \multirow{2}{*}{$\begin{array}{l}\text { Particle no. } \\
\left|m_{v}^{\text {int }}\right|(\mathrm{meV})\end{array}$} & \pm 1 & $0 / 2$ & 3 & 4 \\
\hline & 9.09 & 5.82 & 0.0880 & 0.0656 \\
\hline
\end{tabular}

\section{Conclusion}

We proposed the internal constituent motion of electron neutrino, which was taken to be consistent with theU(1) $\times \operatorname{SU}(2)$ frame of $\sin ^{2} \theta_{W}=0.231$. The imposition of momentum transformation function to gamma matrices explained the existence of reversed type of auxiliary field. The field creates the constituent kinetic mass, which equals the spin magnetic energy. The mass was utilized in potential propagator, and was considered to work as a potential creating boson. Its product with Lagrange multipliers explained the heavy weak boson mass, like $\mathrm{Z}^{0}$. Numerical calculation demonstrated oscillation length around $100 \mathrm{~km}$ with two forward and backward motions around the center of mass. Discussion was made on possibility of creation additiojmhy6nal constituent with V-type spin. Such neutrino may exist in nature as environmental neutrino, and readily causes additional reaction with other materials.

\section{Acknowledgements}

The authors express our gratitude to Prof. N. Ikeda and Prof. K. Maehata of Kyushu University for cooperation for the study.

\section{References}

[1] S.L. Glashow, Partial-symmetries of weak interactions, Nucl. Phys. 22 (1961), pp. 579-588.

[2] S. Weinberg, A model of Lepton, Phys. Rev. Lett. 19 (1967), pp. 1264-1266.

[3] A. Salam, Weak and electromagnetic interactions, 8th Nobel Symp. Lerum, Sweden, May 19-25, 1968, (1968).

[4] P. Higgs, Broken Symmetries and the masses of gauge bosons, Phys. Rev. Lett. 13 (1964), pp. 508-509.

[5] T.W.B. Kibble, Symmetry breaking in non-Abelian gauge theories, Phys. Rev. 155 (1967), pp. 1554-1561.

[6] K. Hagiwara, et al. (Particle Data Group), Review of particle properties, Phys. Rev. D66 (2002), p. 010001 .

[7] B. Pontecorvo, Nuclear capture of mesons and the meson decay, Phys. Rev. 72 (1947) pp. 246-247.

[8] Z. Maki, M. Nakagawa and S. Sakata, Remarks on the unified model of elementary particles, Prog. Theor. Phys. 18 (1962), pp. 870-880.

[9] M.C. Gonzalez-Garcia, M. Maltoni and T. Schwetz, Nucl. Phys. B 908 (2016), pp. 199-217.

[10]S. Suda, et al., PWR reactor irradiation effects on the electrochemical device with use of biological product, 9th Int'l Symp. on Radiation Safety and Detection Technology (ISORD-9), Nagoya, Japan, July 10-14, 2017, (2017).

[11]N. Nakanishi, Covariant quantization of the electromagnetic field in the Landau gauge, Prog. Theor. Phys. 35 (1966), pp. 1111-1116. 